\title{
Politique
}

\section{Crise de l'État-Providence : idéologies, réalités et enjeux dans la France des années 1980}

\section{Alain Lipietz}

Numéro 4, automne 1983

Crise et changements idéologiques

URI : https://id.erudit.org/iderudit/041002ar

DOI : https://doi.org/10.7202/041002ar

Aller au sommaire du numéro

Éditeur(s)

Société québécoise de science politique

ISSN

0711-608X (imprimé)

1918-6584 (numérique)

Découvrir la revue

Citer cet article

Lipietz, A. (1983). Crise de l'État-Providence : idéologies, réalités et enjeux dans la France des années 1980. Politique, (4), 55-103.

https://doi.org/10.7202/041002ar d'utilisation que vous pouvez consulter en ligne.

https://apropos.erudit.org/fr/usagers/politique-dutilisation/ 


\title{
Crise de l'État-Providence: idéologies, réalités et enjeux dans la France des années 1980
}

\author{
Alain LIPIETZ. \\ Centre national de la recherche \\ scientifique
}

En France, comme dans toute l'OCDE, l'État-Providence est en crise. Crise qui va de pair avec celle de l'État-Keynésien, mais crise bien distincte. D'ailleurs, le Keynes «text-book» n'aurait jamais osé suggérer l'État-Providence tel qu'il s'est développé ${ }^{1}$. Pourtant, les dépenses de l'État-Providence ne constituent qu'une partie de la «demande effective» dont Keynes prônait la croissance. Mais en fait, l'État-Providence est avant tout victime de la non-croissance, alors que les politiques keynésiennes sont condamnées pour leur incapacité à susciter cette croissance. Sa crise est objective tout autant que subjective.

Soyons donc précis et nets. «L'État-Providence» désignera ces institutions, placées sous la tutelle de l'État, qui assurent la prise en charge monétaire des risques et des besoins nés des aléas de la vie humaine: éducation de jeunes enfants, maladies, accidents, vieillesse, et aujourd'hui chômage. Nous en exclurons

1. Keynes, en 1926, estimait impossible «d'un point de vue politique» que les dépenses publiques puissent atteindre le quart du revenu national (ROSANVALLON [1981]). Aujourd'hui on en est à 40-60\% selon les pays de l'OCDE. 
donc l'intervention de l'État dans le financement des besoins permanents, dont l'éducation et le logement, qui font pourtant intégralement partie de ce qui sera pour nous à la racine de l'État-Providence ${ }^{2}$ : la gestion collective de la reproduction de la force de travail. Cela fait déjà beaucoup, et c'est très cher. De plus en plus cher, et c'est cela la seule crise objective de l'ÉtatProvidence: on ne sait plus comment le payer.

Alors, bien sûr, on le remet en cause. Mais cette «crise de légitimité" n'en est une que pour les intellectuels organiques, ceux qui ont la charge de «penser» la crise. Certes, on objectera la révolte des contribuables californiens, la «désertion» d'un Bergman ou d'un Borg, etc... Que ceux qui sont appelés à payer de lourdes contributions obligatoires aient tendance à frauder ou à «voter avec leurs pieds» est une évidence qui n'a pas attendu la crise particulière de l'État-providence pour se manifester. Toute la question est de savoir si, de toutes les dépenses organisées par la puissance publique, l'État-Providence (pour nous à présent réduit à la Protection Sociale obligatoire, qui se résume institutionnellement en France à la Sécurité Sociale et à ce petit rejeton tardif mais plein d'avenir, l'Assurance-chômage) est le plus exposé à la vindicte populaire (par rapport aux dépenses d'armements, par exemple). Or la réponse est nettement, et de plus en plus, négative, du moins en France.

Un sondage, publié dans le mensuel L'Expansion (6 Mai 1982), est particulièrement éclairant. À la question, «Pour chacune des choses suivantes, diriez-vous, si on la supprimait, que ce serait grave?», les sondés consacrent à la quasi unanimité la Sécurité Sociale comme la vache la plus sacrée. Très grave: $85 \%$, assez grave: $12 \%$, pas grave ou pas d'opinion: $1 \%$ chacun. «Très grave» recueillait $79 \%$ en 1976 . À titre de compa-

2. Voir l'étude magistrale de DELORME-ANDRÉ [1982] sur l'ensemble des dépenses publiques. 
raison, la suppression du droit de vote serait jugée «très grave» par $81 \%$, de la liberté de la presse par $70 \%$, du droit de grève par $49 \%$, et des partis par 33\%... Malheureusement, remarque L'Expansion, «il est préoccupant de constater que $31 \%$ seulement des citoyens estiment qu'un bon citoyen ne doit pas frauder le fisc » (les électeurs du P.S. étant de cet avis à $28 \%$, ceux du PC à $34 \%$ !). Tout est là.

Tout est là, mais peu de parents sont prêts à mettre leurs enfants sur la paille pour aller mener la grande vie. On peut supposer qu'il existe une corrélation entre la motivation à payer et la clarté du rapport entre ce qu'on paye et les objectifs annoncés. Aux USA, la couverture du risque maladie est largement privée: et les Américains consomment - et donc paient librement - plus de soins de santé que les Français. De même, la cotisation retraite y est pour une large partie non obligatoire, et pourtant une majorité, selon les sondages, affirment préférer voire croître leurs cotisations que décroître leurs futures pensions de retraite ${ }^{3}$.

C'est parce qu'il y a une crise financière de la Sécurité Sociale que se pose nécessairement, à travers le problème de son financement, le problème de sa légitimité. La bataille idéologique est donc seconde à la bataille économique. Mais, puisque la valeur ajoutée n'est pas extensible, il faudra bien choisir entre revenus directs, revenus des transferts sociaux, investissements et autres dépenses publiques. C'est par le biais de cette nécessité de choisir que s'introduit le débat (apparemment sans pertinence pour «l'opinion publique» telle que la saisit les sondages) sur la légitimité de l'État-Providence, Et ce débat s'appuie sur des tendances réelles de la Société.

Nous commencerons donc par un bref examen de ce débat, tel qu'il se déroule aujourd'hui en France. Puis nous examine-

3. Business Week, 29 Novembre 1982. 
rons la fonction et la crise de la protection sociale dans la réalité de l'expérience française. Nous proposerons pour finir quelques pistes pour une solution «progressiste» à la crise actuelle.

\section{Le débat}

Pour simplifier, nous nous limiterons à un échantillonnage restreint, presque caricatural, des succès de librairie sur la scène idéologique française (qui prend aujourd'hui la forme étrange du «pamphlet savant» ${ }^{4}$ ). Pour achever le style caricatural, nous classerons les positions de droite à gauche. Les étiquettes ainsi attribuées relèvent purement de l'ordre d'exposition du présent texte et ne mesurent pas les «distances politiques» réelles sur la scène politique française. Pierre Rosanvallon s'estimera peutêtre vexé d'un qualificatif de «centre-gauche» qui le situera trop près du "centre-droitier» Henri Lepage. Le lecteur de cet article notera en outre que la place «gauche» est manquante et en déduira, non sans raison, que, conformément à une tradition bien ancrée dans la France d'Après-Guerre, l'auteur entend l'occuper lui-même.

\section{À droite: Pbilippe Bénéton}

L'auteur du «Fléau du bien [1983], comme Henri Lepage que nous visiterons ensuite, s'inscrit précisément dans la réaction tardive à la marque indélébile qui frappa la droite française

4. Le "pamphlet savant" est un livre qui vise à développer une idée qui autrefois se serait contentée d'un article de revue ou d'un court libelle. La restructuration de la scène idéologique par les médias de masse condamne aujourd'hui ces formes d'expression: ni un article isolé dans une revue, ni un libelle ne peuvent justifier une campagne publicitaire. Le "pamphlet savant» est donc un pamphlet gonflé jusqu”a la taille d'un livre respectable en volume, par incorporation des résultats d'études savantes, les références mathématiques, historiques ou philosophies, incontrolables par un public de masse, étant censées renforcer le poids de la thèse. Tous les livres que nous allons citer ne se conforment évidemment pas à ce schéma: il y a loin du dinosaure de Bénéton à l'oiseau de paradis d'André Gorz. 
à l'issue de sa honteuse collaboration avec le nazisme. La «Nouvelle philosophie» ayant réussi, au milieu des années 70 , à sataniser le marxisme à la suite du stalinisme, on vit fleurir dans les maisons d'édition des collections où s'affirmèrent bien haut les convictions libérales-conservatrices: au nom des libertés et du pluralisme, les intellectuels de droite prenaient enfin leur revanche sur l'idéologie «étatiste» du Conseil National de la Résistance (qui incluait le Gaullisme, mais non la famille politique du Président Giscard d'Estaing, au pouvoir de 1974 à 1981). Mais, le terrain ayant été laissé en friche pendant trente ans, l'extrémisme libéral pouvait sans pudeur se targuer de l'étiquette "Nouveaux économistes» (alors même qu'ils s'inscrivaient dans le droit fil de l'enseignement néo-classique qui n'avait jamais perdu le monopole des seules institutions dignes d'intérêt: les Grandes Écoles), et compenser leur «nouveauté » (donc la fraîcheur de leur ignorance de la société française) par la référence à une base d'appui solide: l'immense massif anglosaxon.

On trouvera donc dans ces ouvrages peu d'éléments nouveaux par rapport à l'apport théorique anglo-saxon, et presqu'aucune analyse concrète de la réalité française.

$\mathrm{Au}$ sein du massif libéral, Ph. Bénéton représente sans doute la droite. Son livre, sous-titré «Essai sur les politiques sociales occidentales», ne parle quasiment que des U.S.A. Mais le diagnostic est très imple et de portée générale (chapitre VIII): il y a trop de redistribution.

Économiquement, on diminue ainsi la propension marginale à travailler, et on accroît le bénéfice marginal du loisir. Conséquence: la «désertion» devant le travail, qui prend essentiellement deux formes: la «paresse» et, pour ceux qui s'obstinent à travailler, «la fraude». Ajoutons en outre que la redistribution est un jeu à somme négative (puisqu'il faut payer la bureaucratie redistributrice). Remède: à chacun les revenus 
directs de son seul travail, et les noirs fainéants reprendront le chemin de l'usine, les dentistes suédois le chemin de leur cabinet. Corollaire: il faut condamner les notions de « revenu minimum garanti » et autres «impôts négatifs» versés aux indigents, car de deux choses l'une,

- ou cette assistance est coupée dès lors que l'indigent se met à travailler, et il ne gagne rien à se remettre au travail;

- ou la suppression de l'assistance est amortie jusqu'à la zone des revenus moyens, et son coût est prohibitif. "Sauf à fixer le revenu garanti très bas, le dilemme de l'impôt négatif n'a pas de solution » (p. 171).

Opinion corroborée par Jean-Dominique LAFAY [1983], qui paraphrase, selon l'habitude de ce courant, une étude anglo-saxonne (PARKER [1982]). Celle-ci rapporte, au salaire moyen net de prélèvement d'un ouvrier masculin, le revenu d'un ménage ne travaillant pas, pour définir un «taux de remplacement en termes de pouvoir d'achat». Ce taux est passé, en Grande Bretagne, pour un couple marié, avec deux enfants, de $46 \%$ en 1948 à $71 \%$ en 1981 , et, avec quatre enfants, il atteint $94 \%$ en 1981 . Pourquoi travailler?

Évidemment, on peut s'interroger sur la signification de tels chiffres. Remarquant que le taux n'est que de $33 \%$ pour un célibataire, on en déduit qu'une majoration familiale existe. Ou bien ces allocations familiales sont maintenues pour un travailleur, et c'est abusivement que l'auteur néglige de les compter dans les revenus disponibles, ou bien elles ne le sont pas, et c'est une absurdité de la législation sociale anglaise que peut modifier un simple vote. Plus profondément, ce réductionnisme fait fi des études sur les mobiles réels du travail salarié, qui poussent vers celui-ci y compris les femmes dont le conjoint travaille, (LINHART [1981]), et sur les cercles vicieux qui refoulent le «quart monde» dans l'assistance, loin du travail régulier. 
En tout état de cause, la critique de droite à la Bénéton n'a, pour le moment, aucun écho en France, si ce n'est sur la question bien circonscrite de la date et du montant des « fins de droits» pour les chômeurs. La légitimité de la «solidarité» à l'égard de ceux qui ne peuvent subvenir à leur besoin est acquise depuis l'Ancien Régime, le progrès social en a fait un droit, et non plus une irresponsable libéralité de la charité. Reste la critique de l'inefficience: et c'est celle de Lepage.

Au Centre-droit: H. Lepage.

Contrairement à Bénéton, $\mathrm{H}$. Lepage, tout en se réclamant du conservatisme, concilie un vigoureux appel au libéralisme avec un faible pour une charité cuirassée d'intérêt bien compris. Le secours aux nécessiteux lui paraît légitime: avec l'ancien ministre giscardien L. STOLERU [1977], il reconnaît que la société libérale-démocratique se doit de «vaincre la pauvreté dans les pays riches». Il est donc partisan de l'assistance sous forme d'impôt négatif. D'ailleurs, sur les 448 pages de Demain le capitalisme [1978], hymne aux vertus du marché généralisé et anathème contre l'intervention et la réglementation publiques, il ne consacre que 8 pages à la critique de la protection sociale, et 6 pages sur les 576 pages de Demain le Libéralisme [1980].

Et que dit-il? Qu'il n'y a pas assez de redistribution. Le reproche ne vise pas l'intention, mais l'inefficience. Brassant des centaines de milliards (tout en en prélevant des dizaines), le système redistribue indéfniment l'argent des «classes moyennes" dans un jeu à somme négative. Constat peu original sur lequel Lepage ne donne aucun chiffre (pas même pour préciser l'ampleur des "classes moyennes»). En revanche il applique la théorie des "choix publics" à l'explication de ce phénomène.

Tout d'abord, le caractère public de cette redistribution est rapporté, comme il se doit en individualisme méthodologique, à 
la stratégie des agents. La charité est une satisfaction, la paix sociale aussi. Mais ces besoins sont plus économiquement satisfaits sous la forme de «biens collectifs» publics. «La redistribution; une assurance anti-conflictuelle... Une explication qui malgré son évidence n’a jamais été citée dans les manuels, que ceux-ci soient néo-classiques ou marxistes» (p. 181).

Passons sur la légèreté de la dernière affirmation: s'il est une explication «triviale» de la protection sociale chez les marxistes, c'est bien celle de «l'armistice sociale», le patronat et son État concèdant durablement les seules «conquêtes ouvrières" qui lui valent la paix. Nous n'aurons nous-mêmes pas grand chose à en dire de plus, si ce n'est qu'elles lui ont aussi valu la croissance. Mais Lepage va plus loin et cherche à expliquer pourquoi ce transfert net minime en faveur des plus défavorisés mobilise un brassage qui atteint la quasi totalité de la population. C'est pour lui un effet du paradoxe d'Hotteling sur le marché des décisions politiques en démocratie: la minorité la plus pauvre ne peut l'obtenir que si elle rallie le centre en une coalition dont l'enjeu est le repartage général.

Explication non sans pertinence, un peu légère dans sa formulation: de fait, les classes moyennes se sont toujours battues pour bénéficier des avantages consentis aux couches plus défavorisées. Mais ce qui étonne, c'est la nécessité d'une explication. Car enfin, les pères de l'État-Providence, les forces sociales qui l'ont imposé, les forces politiques qui l'ont voté, n'ont jamais prétendu en faire un appareil de transfert massif d'une tranche de revenu à une autre (à l'exception des plus pauvres, qui relèvent de l'assistance budgétaire, et non de la Sécurité Sociale d'ailleurs). Si «compensation» et «transfert» il y a, c'est à l'intérieur des différentes tranches ou catégories socio-professionnelles, entre les individus qui ont vocation à être normalement insérés dans les circuits de reproduction marchande-capitaliste, et vers ceux d'entre eux qui, à un moment donné de leur vie, ne peuvent pas 
(maladie, chômage), ou ne peuvent plus (retraite) percevoir normalement leur «revenu permanent», ou ont transitoirement à charge des dépenses liées à la satisfaction du devoir national de natalité. La protection sociale combat la pauvreté du malade, du chômeur ou du vieux, non l'inégalité statistique dont souffre la classe ouvrière par rapport aux classes possédantes.

Comme nous le verrons, la Protection sociale vise fondamentalement à régulariser le revenu des différentes classes sociales, et centralement le rapport salarial, non à remettre en cause le partage du revenu national. Sa crise véritable naît donc de la nécessité d'une modification des normes de ce partage. C'est ce que voit bien Alain Minc.

Au centre: Alain Minc.

Avec ce très jeune et très brillant Inspecteur des finances, né en 1949, auquel la rumeur prête un passé «gauchiste», promu à la direction du Groupe St Gobain-Pont-à-Mousson avant sa nationalisation (il en est aujourd'hui directeur financier), la parole revient non à un idéologue abstrait, mais à une incarnation étonnante du «fonctionnariat du capital anonyme» (quoique nettement français). Plus de supputations sur les fondements psychologistes du marché généralisé, mais, sur la base d'une analyse concrète, la froide contrainte du paiement au comptant.

Les "nouveaux économistes», tenants de la théorie de l'utilité-rareté, réduisent, on le sait, l'économie à la psychologie (dans la plus complète ignorance des évolutions de cette dernière, telle la psychanalyse). Expliquant le réel par des fonctions (ou ensembles) de préférences non explicitées dans la tête des individus, ils devront tôt ou tard se rallier à "l'axiomatique de la préférence révélée" - révélée par le réel ${ }^{5}$. Au nom de quoi dès

5. Les «nouveaux économistes» évoquent parfois les «effets pervers». Mais comment savent-ils qu'ils sont "pervers»? Et si le but réel et tacire de la coalition 
lors critiquer un état de fait unanimement accepté (par dela les grincements de dents, naturels dès qu'il s'agit de payer quoi que ce soit)?

Avec Alain Minc, le verdict est clair: qu'on le veuille ou non, il n'y a plus de quoi payer. Conformément aux règles du pamphlet savant, Minc pose initialement deux «contraintes», puis promène le lecteur avec compétence et point trop de grâce dans les débats actuels de prospective industrielle internationale et de sociologie politique, puis suggère en quelques pages sa réponse aux contraintes. Contrainte majeure: la croissance est durablement cassée à l'échelle mondiale, et l'imbrication des économies interdit à chaque pays, sous peine de déficit dramatique, de «relancer tout seul». Contrainte mineure: «l'incompatibilité de l'État-Providence tel qu'il fonctionne et d'une économie sans croissance». On apprendra peu de chose des raisons de la crise mondiale, on n'étudiera pas les possibilités de relance concertée, on n'entendra pas parler du Tiers-Monde, mais on dégonflera au passage la baudruche de l'issue «électronique» à la crise : «une révolution sans miracle», qui ne fera qu'accroître la concurrence au sein d'une économie-monde globalement stagnante.

D'où l'impératif: réorienter les fonds vers la modernisation industrielle, seul l'État pouvant prendre la direction de cette gigantesque opération de sauvetage. Non en gérant lui même, mais en bâtissant un «bouclier protecteur» pour l'industrie, le fer de lance devant être des entreprises nationalisées érigées en féodalité à la japonaise. Il faut «davantage d'État car le marché économique mondial n'est pas la bluette imaginée par les économistes libéraux du $\mathrm{XIX}^{\mathrm{e}}$ siècle. C'est désormais un champ de bataille où s'affrontent des forces qui s'affranchissent du jeu tra-

mäjoritaire qui vote l'assustance chômage était justement d'exclure une partie de la population du marché du travail (thèse du « vétéro-machisme» de Michel Albert [1982]) et de fournir à la petite bourgeoisie intellectuelle des emplois de bureaucrates sociaux? 
ditionnel de la concurrence parfaite. Chacun ruse avec le marché pour y survivre. Ruser, c'est en l'occurrence distordre artificiellement les contraintes de rentabilité, privilégier des objectifs à long terme, s'assurer, à n'importe quel prix, une position dominante au cœur de l'économie-monde. Ce sont des actions de longue haleine qui exigent à la fois une intervention régalienne et un transfert de ressources, en un mot une présence de l'État. À lui qui n'était jusqu'alors que redistributeur - l'État-Providence - ou protecteur - l'État régalien -, de s'immerger au cœur de l'économie-monde. Il lui faut être productif sans être producteur» (p. 193).

Mais cela, l'État ne pourra pas le faire s'il se ruine à rester "protecteur». Les normes légales de dépenses de la protection sociale conduisent à prévoir leur progression avec une régularité de métronome: plus de vieux, plus de chômeurs, plus de consommation médicale. En croissance ralentie, ce serait $1 \%$ à $2 \%$ par an de plus à prélever chaque année sur la valeur ajoutée. Une seule solution: «marchandiser l'État protecteur», laisser la «société civile» ${ }^{6}$ se débrouiller. Par l'autoproduction, la solidarité de voisinage, et s'il faut en passer par des débours marchands, par l'assurance facultative qui pourra d'ailleurs conserver un statut de droit public: pas plus question de «tondre des coupons» sur le dos du capital des assurances que sur celui du capital industriel.

Derrière la plaidoierie exaltant l'inventivité de la société civile, opposée aux mécanismes administratifs, l'argument macroéconomique est très simple: la population ne peut plus se voir garantir la croissance des revenus de transfert. $\grave{A}$ total des revenus directs et indirects donné, chacun choisira d'être cigale ou fourmi - mais le surplus net est réservé à l'industrie.

6. Concept que Minc ne définit jamais de façon cohérente. Il ne faut l'entendre ni dans son acception classique, ni dans son acception gramsciste. C'est en gros, chez Minc, la flexibilité et l'esprit d'entreprise des individus. 
Si A. Minc est bien au centre de notre échantillon, c'est un centre «hyper-moderne», tout à fait caractéristique des mutations en cours dans l'idéologie française, précipitées par la victoire du Parti Socialiste. À la vieille récrimination de gauche contre l'usage droitier du secteur public ( «la socialisation des pertes et la privatisation des profits»), il oppose le mot d'ordre du capitalisme d'État: «Nationalisation des profits et privatisation des coûts». Et ce faisant résoud son problème de directeur financier.

Malheureusement son projet (il est vrai laissé à l'état d'ébauche) ne répond pas à l'impératif macroéconomique qui a sous-tendu la reproduction de l'État-Providence: régulariser le cycle des revenus privés. Ce n'est pas un hasard si l'assuranceautomobile est restée privée (quoique obligatoire) et l'assurance-maladie devenue publique. Laisser chacun redevenir cigale, c'est briser les régulateurs institutionnels qui avaient garanti la croissance et modéré la crise. Par ailleurs, socialement, les risques sont évidents. Comme le dit X. GREFFE [1983]: «l'assurance, c'est la société duale», la médecine et la retraite à deux vitesses. Mais n'est-ce pas déjà largement le cas?

Au centre-gauche: P. Rosanvallon.

Penseur de la CFDT et chroniqueur de Libération, P. Rosanvallon occupe à ce titre une position de gauche novatrice mais modérée. Il ne partage pas le parti-pris de Minc en faveur du capital, mais, sans vouloir remettre en cause trop radicalement les équilibres entre les classes, il condamne le «socialcorporatisme " ${ }^{7}$ qu'engendre l'État-Providence, et s'interroge sur la légitimité de sa croissance par rapport aux nouvelles aspirations individualitaires.

7. C'est-à-dire la tendance de tous les groupes sociaux, y compris les salariés, à se constituer en clientèle de l'État. 
Brisant un dogme qui tend à s'établir, il remarque d'abord [1981] qu'il n'y a pas de limite théorique à la socialisation du revenu (mais seulement un choix de société sur la «socialisation tolérable») et ajoute à juste titre [1983] que les dépenses de la protection sociale ne sont pas des «charges» prélevées sur la Société. Mais déjà le texte de [1983] admet implicitement que la croissance des revenus indirects ne peut se faire qu'au détriment du revenu direct, ce qui est reconnaître la nécessaire stagnation, dans la crise, du revenu disponible des ménages, sur des arguments assez semblables à ceux d'Alain Minc. Mais sans s'attarder sur l'impératif industriel, P. Rosanvallon s'étend au contraire longuement sur la critique du «tout à l'État».

Pour ce faire il présente d'abord sa propre analyse de l'État-Providence. Il s'agirait d'une simple extension de «l'État-protecteur» caractéristique de l'État moderne, celui qui veille à l'ordre et à la sécurité des biens et des personnes. Dès lors que la Société cesse de se penser comme un corps, mais comme un marché, la protection prend la forme d'une «providence redistributive» fondée sur la possibilité statistique d'équilibrer les aléas individuels. La «providence» étatique exclut la solidarité antérieurement encastrée dans le social, et lui substitue des normes administratives de compensation. Et Rosanvallon de citer régulièrement le Rapport Beveridge (1942) qui fixa pour but à l'État-Providence de «libérer la société du besoin et du risque».

Or ces normes administratives de "couverture» ont deux défauts. Elle aboutissent à compenser des inégalités qui peuvent être perçues comme légitimes (toujours la cigale et la fourmi). Elles entrainent automatiquement, dès que la croissance sociale ralentit, une augmentation de la part socialisée, augmentation mécanique qui n'est pas la contrérartie d'un mouvement politique en faveur d'une solidarité croissante. D'où les « réactions de défense de la Société» que sont le travail noir, la fraude, etc... 
Face à cette délégitimation, non de l'État-Providence, mais de sa croissance mécanique, deux grands axes de solutions:

- «réencastrer» la solidarité dans le social (en court-circuitant l'État),

- revenir à une plus grande "lisibilité» des transferts sociaux, de leurs raisons et de leurs buts.

Le premier conseil (resocialiser la solidarité) n'est pas mauvais, mais $\mathrm{P}$. Rosanvallon semble oublier qu'à l'époque de la «solidarité organique», la charge de la providence retombait sur... les femmes. Mères, épouses, filles aînées et «bonnes sœurs» recueillaient les laissés pour compre de la production marchande, prenaient en charge enfants et vieillards, épongeaient à mains nues la misère du monde. «Mettre les vieux à s'occuper des enfants», c'est en fait y remettre les vieilles. Or le mouvement des femmes met en crise la «société-civile-providence» aussi sûrement que la croissance des cotisations déstabilise l'État-Providence. Cela ne signifie pas que la reprise en charge par les «structures du quotidien» de certains impératifs de la solidarité soit condamnable. Mais cela exige un examen attentif, favorisant une remise en cause radicale de la vieille division sexuelle du travail ${ }^{8}$. Malheureusement sur ce point, comme sur l'immixtion des intéressés eux-mêmes dans la gestion des institutions de l'État-Providence, le débat français est bien en retard par rapport aux réflexions allemandes, québécoises ou anglo-saxonnes qu'illustrent par exemple Rowborham, Segal, Wainwright [1970].

8. D'une façon générale, P. Rosanvallon [1980] sous-estime la sauvagerie des formes d'exploitation dont "l'économie souterraine" est le siège, exploitation des femmes, des enfants, ou tout simplement exploitation capitaliste "non-déclarée", fraudant la Sécurité Sociale, le fisc, et ignorant la législation du travail, et condamne trop vite la tentation de l'État d'y mettre le ho-là. La CFDT elle-mème en vient à s'inquiéter du projet de J. J. DUPEYROUX [1983] de donner aux victimes de cette exploitation le droit de porter plainte contre les organisateurs du travail noir! La haine de l'Étatprotecteur fait de tels ravages qu'on en vient même à gauche à parler de «délation" pour désigner l'activité minimale des délégués syndicaux: faire respecter le droit... 
Quant au premier conseil (accroître la transparence), il est excellent. On constatera alors que l'État-Providence, loin de relever de l'organisation d'une société de citoyens, comme le pense Rosanvallon, relève de la régulation d'une société de classe, qu'il contribue à reproduire. Nous y reviendrons.

En Utopie: André Gorz.

Absolument symétrique de Minc par rapport à Rosanvallon, André Gorz pousse à l'extrême l'exaltation de la créativité de la «société civile», et nie purement et simplement le problème des coûts de la protection sociale. Si dans son dernier livre [1983], il n'affirme plus que la crise soit née d'un excès de productivité, il maintient, comme en [1980], que les gains prodigieux de productivité entraînés par l'automatisation interdisent à l'avenir une reprise des rapports marchands traditionnels, et accomplissent la prophétie de Marx: l'abolition du règne du besoin et de la rareté, donc de la valeur-travail et du capital. La "production hétéronome» peut satisfaire à tous les besoins «lourds» et assurer un revenu permanent garanti, moyennant une dépense minime de "travail obligé" (20000 heures par vie) dûe à la société. Tout le reste du temps peut être consacré à l'amour, à la création, au loisir: aux activités autonomes ${ }^{9}$.

Ainsi, la critique radicale de l'État-Providence débouche sur le comble de l'État-Providence: le revenu garanti. Mais ce n'est plus une "providence», et il ne compense plus l'absence anormale de revenu d'activité. C'est un droit régulier à « revenu social», "qui n'est plus assis sur la valeur du travail ni conçu comme une rémunération de l'effort. Il a pour fonction essentielle de distribuer sur tous les membres de la société une ri-

9. Ayant subi de rudes critiques de la part des féministes lors de la parution de l'ouvrage de [1980], A. Gorz prend maintenant bien soin de souligner que l'exploitation domestique n'a rien à voir avec l'activité autonome. 
chesse résultant des forces productives de la société dans son ensemble» [1983, p. 91]. Ce revenu rémunère le citoyen en tant qu'être social, un point c'est tout. « Rémunération et prestation de travail sont déconnectées».

Inutile d'insister sur le caractère «utopique» d'un tel projet. D'abord parce que GORZ finira sans doute par admettre que le problème actuel reste (entre autres) l'insuffisance des gains de productivité, non leur excès (BOYER, MISTRAL [1983]). Ensuite parce que GORZ reconnait, au moins par intermittence, que ce parachèvement du revenu garanti suppose une révolution sociale. Il est plus intéressant de souligner que son livre recoupe non seulement des aspirations authentiquement progressistes et des pratiques qui tendent à se répandre dans une minorité «décalée» (travailler un minimum pour assurer ses droits aux revenus de transfert, et faire des choses intéressantes à côté), mais également des tendances qui se dessinent dans certains secteurs «hyper-modernes», à découpler une bonne fois le problème du droit au revenu de celui de l'offre d'emploi salarié.

En témoigne le livre de Michel ALBERT [1982], qu'on pourrait qualifier politiquement de «centriste», et qui pourtant propose tout simplement, après la couverture de la maladie, du chômage et de la retraite, une nouvelle extension de l'ÉtatProvidence: la couverture du travail à mi-temps, moyennant un nouveau bond en avant des prélèvements obligatoires, égal à $25 \%$ de l'actuel impôt sur le revenu. Partant du constat que l'offre d'emploi ne peut plus joindre la demande, il faut qu'un grand nombre $(30 \%)$ accepte de travailler à temps partiel pour que tous puissent travailler (à un «volant frictionnel» de 960000 chômeurs près!). Mais si la réduction du revenu était proportionnelle à la réduction de l'activité, il n'y aurait guère de volontaires. L'État prendra donc à sa charge, à titre de compensation ( $40 \%$ du salaire), une «prime aux Volontaires du travail à Temps Réduit. 
Rappelons que Michel Albert fut le Commissaire du Plan de Raymond Barre, et dirige actuellement une importante compagnie d'assurances. On constate que les anathèmes des nouveaux Économistes, les contraintes d'Alain Minc et les prudences de P. Rosanvallon contre l'extension de l'État-Providence ne font pas l'unanimité dans les classes dirigeantes françaises...

\section{Une analyse en terme de régulation}

Toutes les positions dont nous venons de parler ont un point commun. Elles opposent d'abord des citoyens marchands (ou auto-producteurs) à l'État. Dans ce couple citoyen - État, le débat porte sur le degré de socialisation du revenu monétaire que suggèrent la justice distributive, la nécessité, l'efficience, etc..., du $\mathrm{O}$ (chez Bénéton) à l'infini (chez Gorz). P. Rosanvallon est le plus conséquent dans l'approche «philosophie politique et Bénéton dans la problématique «économiste». Mais le couple reste un invariant.

Or, selon le mot célèbre de Marx, «la Société n'est pas composée d'individus». La conception même de l'individu en position de citoyen-échangeur est le produit de la généralisation des rapports marchands (DUMONT [1977]). Mais ces rapports ne sont pas les seuls. Outre les rapports domestiques et « informels», abondamment invoqués dans les contributions citées plus haut, nos sociétés capitalistes sont structurées par le rapport salarial. Ce rapport présuppose les rapports marchands et en emprunte les formes, mais ne s'y réduit en aucune manière ${ }^{10}$. Il est totalement impossible de comprendre le développement de la Protection Sociale en faisant abstraction de ce rapport. La démarche matérialiste doit partir de cette réalité sociale, et montrer d'une part le processus historique et, de l'autre, la logique

10. Voir l'analyse des rapports constitutifs du mode de production capitaliste dans LIPIETZ [1979]. 
du développement de la Protection sociale dans les sociétés capitalistes.

Il y a en effet deux manières, complémentaires, d'introduire le rapport salarial. D'une part, l'approche généalogique, diachronique. On montre alors comment la lutte des classes entre ouvriers et patrons «invente» les embryons de la protection sociale, les «compromis institutionnalisés » qui, se généralisant ensuite au reste du salariat, puis aux autres classes sociales, constituent progressivement les dispositifs de l'État-Providence. C'est la voie privilégiée par R. Delorme et C. André [1983]. D'autre part, il y a l'approche structurale, synchronique. On étudie alors quel rôle joue à telle époque, dans tel régime d'accumulation, l'existence d'une telle institution, dans la régulation, c'est-à-dire la résolution des contradictions, du rapport salarial"'.

Ces deux voies sont rigoureusement complémentaires, comme le remarquent DELORME et ANDRE (p. 653 sq.). Déduire des nécessités de la régulation, l'émergence de luttes de classes, aboutissant à l'institutionnalisation de ce dont le capital «a besoin», serait tomber dans un «fonctionnalisme du pire», un «machiavélisme des structures», indûment prêté aux théoriciens de la régulation, et d'ailleurs dénoncé par ceux-ci ${ }^{12}$. En revanche, tous les «acquis» de la lutte des classes ne sont pas stables, ils ne le deviennent que s'ils parviennent à s'insérer dans un mode de régulation d'ensemble compatible avec le régime d'accumulation. La configuration d'un certain nombre de formes institutionnelles régulatrices et d'un régime d'accumulation est une «trouvaille» historique même si l'intentionnalité n'en

11. La distinction de différents « régimes d'accumulation" et «modes de régulation» et leur couplage a été introduite par le rapport CEPREMAP [1977] dont sont issus BOYER, MISTRAL, LIPIETZ [1979], etc...

12. Voir par exemple ma réflexion [1982] sur la structure des rapports internationaux en termes de «configurations» et de «trouvailles». 
est pas toujours exclue, et si la diffusion d'un pays à l'autre s'appuie sur les succès déjà obtenus.

Nous allons donc présenter très schématiquement ces deux approches, en commençant par l'approche structurale. Elle est en effet de portée générale, et transposable à tous les pays qui se sont trouvés avoir adopté, après la Seconde Guerre Mondiale, le même type de régulation. Puis nous reviendrons sur le processus concret qui, en France, a conduit à cette forme du rapport salarial. Nous déboucherons alors sur l'analyse, dans le cadre de la théorie de la régulation, de la crise financière de l'ÉtatProvidence.

\section{L'État-Providence dans la régulation monopoliste du rapport salarial ${ }^{13}$.}

Dans le rapport marchand, des unités économiques séparées les unes des autres au sein de la division du travail échangent entre elles leurs produits par le biais de la monnaie. La vente des marchandises $(M \rightarrow A)$ sanctionne d'une part la validité sociale du travail engagé dans leur production (c'est la "réalisation»), et d'autre part ouvre un droit (le "pouvoir d'achat» de A) sur la production des autres unités.

Sous la forme d'un contrat marchand, le rapport salarial est tout différent. En échange de la mise à la disposition de sa force de travail $F$, le salarié reçoit du capitaliste une quantité d'argent (le «capital variable V) qui lui permet de se reproduire (à travers les rapports domestiques) en consommant des produits des unités de production capitaliste $(\mathrm{V} \rightarrow \mathrm{M})$. On sait que cet échange laisse au capitaliste un surplus (la plus-value) qui est pour lui le «but» de l'entrée en rapport. Mais ici on s'intéresse à la reproduction du rapport. Comme on le voit, elle suppose de «vrais» rapports marchands (les marchandises capitalistes sont achetées

13. Je reprends ici certains développements de LIPIETZ [1979]. 
en tant que telles par les salariés et les autres capitalistes) et en outre elle prend la forme d'un rapport marchand (la vente de la force de travail $\mathrm{F} \rightarrow \mathrm{V}$ ). La suite des opérations sur le "marché des produits» d'une part, sur le «marché du travail» de l'autre, réalise la reproduction du rapport selon le principe bien connu du «double moulinet»: sorti de l'entreprise avec son salaire, le salarié n'a bientôt plus que sa force de travail à vendre pour recommencer à consommer, et, sorti de l'entreprise avec ses produits, le capitaliste retrouve un «chiffre d'affaire» qui lui permet d'embaucher à nouveau.

Le procès de production capitaliste reproduit donc de lui-même la séparation entre travailleur et conditions du travail. Il reproduit et éternise par cela même les conditions qui forcent l'ouvrier à se vendre pour vivre, et mettent le capitaliste en état de l'acheter pour s'enrichir. Ce n'est plus le hasard qui les place en face l'un de l'autre sur le marché comme vendeur et acheteur. C'est le double moulinet du procès lui-même qui rejette toujours le premier sur le marché comme vendeur de sa force de travail et transforme son produit toujours en moyen d'achat pour le second. Le travailleur appartient en fait à la classe capitaliste, avant de se vendre à un capitaliste individuel. Sa servitude économique est moyennée et en même temps dissimulée par le renouvellement périodique de cet acte de vente, par la fiction du libre contrat, par le changement des maitres individuels et par les oscillations des prix de marché du travail. (Marx [1867, chap. 23, p. 417]).

Les flèches en traits pleins de la figure 1 symbolisent ce «double moulinet», auquel il faudrait ajouter une troisième boucle: les échanges des capitalistes entre eux.

Tout irait pour le mieux si toute chose offerte sur le marché était achetée. Or il n'y a aucune raison, et c'est le principe même du rapport marchand: la métamorphose de l'argent en marchandise $(A \rightarrow M)$ est de droit, celle de la marchandise en argent $(\mathrm{M} \rightarrow \mathrm{A})$ une «épreuve» : c'est tout le problème de la réalisation, du «saut périlleux» de la marchandise. L'entrepreneur n'est pas sûr de vendre, et pour autant que la reproduction $d u$ rapport salarial prend la forme de la vente individuelle de la force de travail, le salarié n'est pas sûr d'être embauché. 
GRAPHIQUE 1

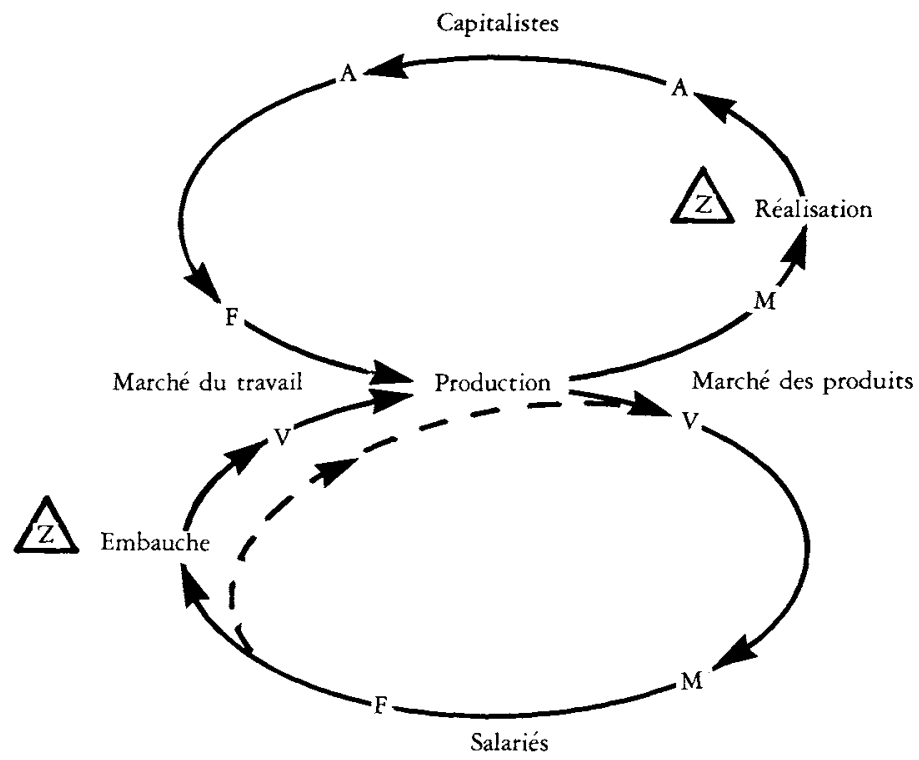

Il peut ne pas l'être parce qu'il est trop vieux, ou malade, ou parce que les entreprises de sa ville ou de sa branche n'ont provisoirement pas besoin de forces de travail (chômage). En outre, pour autant que le salariat comme classe «physique» se reproduit au sein de la famille, il (elle) peut avoir temporairement besoin de s'arrêter de travailler (dans le salariat) pour faire et élever des enfants ${ }^{14}$. Mais alors la métamorphose $\mathrm{F} \rightarrow \mathrm{V}$ n'a plus lieu, ce qui bloque le parcours du demi-moulinet SudOuest $(\mathrm{A} \rightarrow \mathrm{M})$, donc la reproduction domestique $\mathrm{M}-\mathrm{F}$.

14. En outre, les besoins d'argent des ménages salariés varient avec le nombre d'enfants à leur charge, ce dont le rapport salarial d'embauche ne pourrait pas tenir compte s'il était vraiment un pur rapport marchand. 
C'est désastreux pour le salarié, mais aussi facheux pour le capitaliste. D'abord il peut «tenir» à son salarié (qui incorpore un certain savoir professionnel). Surtout, l'insuffisance massive (pour des raisons sur lesquelles nous allons revenir) de la demiboucle $\mathrm{V} \rightarrow$ se traduit pour les capitalistes producteurs de biens de consommation par l'interruption de la demi-boucle Nord Ouest $(M \rightarrow A)$ : on ne vend plus assez. Ce blocage se répercute successivement sur les demi-boucles Nord-Est, (les patrons n'embauchent plus), Sud-Est (d'autres ouvriers entrent en chômage), Sud-Ouest, etc... C'est la «spirale dépressive», la «crise de réalisation».

Ainsi, la forme marchande du rapport salarial est-elle, à elle seule, une cause d'instabilité des trajectoires individuelles des salariés, qui rendrait improbable la reproduction de ceux-ci, sans l'existence d'une «providence» qui, en l'état actuel de nos connaissances sur la bonté divine, ne peut venir que de la Famille ou de l'État, celui-ci s'incarnât-il dans une institution religieuse. En outre, c'est la reproduction d'ensemble du rapport salarial qui s'en trouve fragilisée: la reproduction «par la vente directe» de la force de travail ouvre la possibilité formelle des crises cumulatives.

À cela s'ajoute une source d'instabilité, celle-ci propre au rapport salarial dans sa dimension «subsomption réelle» ${ }^{15}$ : le bouleversement du procès de travail sous la domination du capital accroît la productivité du travail. Le flot de marchandises $M$ sortant du procès de production direct n'a aucune raison de rester adapté à la masse salariale versée $\mathrm{V}$. C'est le risque de crise de surproduction au niveau $\mathrm{V} \rightarrow \mathrm{M}$, qui se répercute sur toutes les autres boucles. D'où le problème de la régulation du rapport salarial, qui est d'abord une régularisation du moulinet inférieur,

15. Ou rapport d' «appropriation réelle» ou de "possession» : la maitrise du procès de travail direct. 
qui est ensuite une adaptation du flux $\mathrm{V}$ au flux $\mathrm{M}$, du salaire direct à la productivité.

Nous voyons ici se dessiner deux variantes de la régulation capitaliste selon une périodisation intimement liée au développement des forces productives:

- une première forme, que l'on peut appeler concurrentielle, de validation au coup par coup de chaque productionreproduction privée, le parcours, d'étape en étape, des demi-boucles.

- une seconde forme, que l'on peut caractériser comme monopoliste, tendant à réaliser, dans l'espace de la circulation, un prolongement des caractères de régularité et de proportionnalité a priori qui sont le propre de la division manufacturière du travail.

Au stade actuel du raisonnement, nous avons relevé ce que doit être une caractéristique décisive d'une telle variante: en ce qui concerne le salariat, cela ne peut signifier qu'une autonomisation du revenu - et de sa dépense - par rapport à la vente individuelle de la force de travail.

Le moulinet inférieur (la reproduction marchande des salariés) ne doit plus pouvoir être interrompu au niveau de l'entrée dans le processus de production: le non-travailleur provisoire doit rester un consommateur. Il faut un "shunt», une dérivation (flèche $\rightarrow$ ) qui saute l'étape de l'embauche quand le travailleur est malade, retraité, chômeur, qui gonfle provisoirement son revenu quand il a des enfants à charge, etc... Ce flux dérivé est prélevé sur la masse totale $\mathrm{V}$ du fond salarial (le capital variable social) qui jusqualors était versé au coup par coup aux salariés. Telle est l'essence du "salaire indirect», dont la gestion, d'abord mutualiste, est progressivement transférée sous la tutelle de l'État: c'est le noyau central de l'État-Providence.

À cela s'ajoute bien sûr la possibilité (la nécessité) de régulariser le salaire direct lui-même, et de moduler la croissance de 
son pouvoir d'achat (comme de celle du salaire indirect) en fonction de la productivité. Dès lors apparaît la possibilité d'ouvrir un crédit de moyen ou long terme aux salariés, gagé sur la régularité institutionnalisée de son revenu.

Les formes structurelles de la régulation monopoliste du rapport salarial seront donc: la contractualisation collective du salariat, la généralisation de formes «longues » et faiblement variables de détermination de la norme salariale (mensualisation, conventions collectives, «contrats de progrès»), l'élargissement du salaire indirect perçu indépendamment de la participation à la production, voire l'engagement des dépenses individuelles en fonction du salaire attendu dans l'avenir (crédit à la consommation pour l'«équipement des ménages» : logement, automobiles...).

Ce qui est déterminant dans la mise en cuvre de ces formes structurelles, c'est le changement de la place des produits de l'industrie capitaliste dans la norme de consommation ouvrière. Ce changement renvoie à un changement dans le mode d'accumulation. On ne peut en effet se lancer dans la production de masse si les «doubles sauts périlleux» restent aussi risqués, si la circulation ne s'appuie pas sur une «société de consommation». En hommage à l'inventeur de la Ford $\mathrm{T}$, on appelle «fordisme» cette transformation conjointe des normes de production et de consommation. Tous ces biens ne s'achètent pas avec un salaire versé au jour le jour!

La mise en place, en France, du fordisme, comme régime d'accumulation intensive centré sur la consommation de masse sous régulation monopoliste, ne sera effective qu'après la Seconde Guerre Mondiale. Concentrons-nous sur le salaire indirect, qui réalise, selon l'expression de MEILLASSOUX [1975], « l'intégration capitaliste à titre viager et non horaire du travailleur dans l'économie capitaliste».

Son institutionnalisation, sa généralisation, s'opèrent nécessairement sous la tutelle de l'État, mieux si des embryons se 
développent antérieurement sous l'initiative du patronat ou des associations ouvrières. En réalité, l'État est dès l'origine impliqué dans la mise en forme du rapport salarial et dans la reproduction de la force de travail (S. de BRUNHOFF [1976]). C'est lui qui a institué (loi Le Chapelier, 1791) la forme juridique du contrat salarial privé, affranchi des règles des corporations. Sous cette apparence d'égalité des sujets marchands, c'est lui qui a institué l'inégalité politique de l'ouvrier, l'a assujetti au «livret ouvrier", et édicté que la parole du patron prévaut en cas de contestation ${ }^{16}$. C'est lui qui a confié aux collectivités locales le devoir d'assistance aux laissés-pour-compte. C'est lui qui reconnaîtra enfin aux salariés le bénéfice du caractère particulier du rapport salarial sous sa forme marchande (en reconnaissant le droit de coalition, puis les conventions collectives). C'est lui qui organisera les grandes négociations instituant la Sécurité Sociale, rendra l'affiliation obligatoire, et affirmera de plus en plus sa tutelle sur sa gestion. Rappelons en outre qu'il assumera la solvabilisation du besoin de logement et, plus tôt, l'instruction publique obligatoire et gratuite.

Le salaire indirect, qui ne représentait que $5 \%$ du salaire direct ${ }^{17}$ en 1934 , atteint $35 \%$ dans les années 50 , et se stabilise à $51 \%$ entre 1968 et 1973. Avec la crise, la croissance du salaire direct se brise: comme un stabilisateur automatique, l'assurance chômage prend le relais. Le flux du salaire indirect dépasse $70 \%$ du salaire direct en 1981. Un «filet de sécurité» s'est déployé contre la spirale dépressive de la crise. La politique du gouvernement socialiste de F. Mitterrand, en accélérant les dé-

16. En France le «délit de coalition» a été aboli en 1864, l'égalité juridique entre patrons et ouvriers (sur le chapitre de la bonne foi présumée) établie en 1868, le livret ouvrier supprimé en 1890.

17. Comme on sait, et pour les raisons que nous verrons, I'État-Providence s'est rapidement étendu à tous, non salariés compris. On ne peut malheureusement pas isoler les transferts sociaux à destination du seul salariat. Nous comparons donc le «salaire indirect" de tous les ménages au salaire direct des seuls salariés. 
parts en retraite, en prenant en charge le salaire des jeunes, en accroissant les prestations familiales, consolidera ce filet, bloquant la croissance du chômage alors que celui-ci s'aggrave très rapidement dans les pays de gestion monétariste-libérale. Cela bien entendu au prix de déficits qu'il faut combler par une hausse de prélèvements, c'est-à-dire par une redistribution accrue des revenus. Au prix d'une compression des revenus directs s'accroît le revenu garanti.

\section{Le processus d'émergence de l'État-Providence.}

En France, l'État-Providence eut d'autant plus de mal à se mettre en place que le rapport salarial s'est camouflé plus longtemps derrière le rapport marchand strictement codifié par le Code Napoléon. La Révolution française, réalisée par l'association des rares industriels et d'une nuée de petits propriétaires et artisans face au monde de la féodalité et des corporations, institue un double rapport pur d'intermédiaires: l'individucitoyen-marchand face à l'État d'une part, au marché de l'autre. Le Consulat, l'Empire, la Restauration renforceront le caractère répressif, anti-ouvrier de la législation civile. Certes, la révolution de Février 1848 inscrit à son fronton le droit au travail, mais les ouvriers sont vaincus en Juin. Une nouvelle alliance se scelle entre patrons et petits propriétaires, mais cette fois contre la classe ouvrière. Alliance renouvellée lors de la défaite de la Commune de Paris (1871).

C'est donc dans un pays de «petits producteurs marchands", où la classe ouvrière a subi deux lourdes défaites politico-militaires en 23 ans, que doivent se dégager progressivement les «avantages sociaux». Pour la seconde raison ils seront très lents, et pour la première raison seront très vite généralisés à la masse des citoyens (à commencer par les fonctionnaires) aussitôt qu'arrachés par la classe ouvrière. 
Dans les pays où la croissance du capitalisme, et donc de la classe ouvrière, ne s'embarassent pas d'entraves «petites-bourgeoises » ${ }^{18}$, le processus est beaucoup plus net. En GrandeBretagne, les lois d'assistance (1601-1662-1782) créent très tôt un «État providence terrien» (à l'usage du prolétariat des campagnes) qui sera amendé dans un sens libéral-capitaliste (abandon de la loi du domicile fixe) en 1834. Mais c'est surtout l'Allemagne de Bismark qui, face à la montée des sociauxdémocrates, fonde un véritable «État-social»: de 1883 à 1889 ont été créés une assurance maladie, une assurance accident, une assurance vieillesse, obligatoires, pour les seuls ouvriers, gérées par eux, alimentées par des cotisations employeurs-salariés. La Grande-Bretagne rattrapera cette avance au début du $\mathrm{XX}^{\mathrm{e}}$ siècle.

En France ${ }^{19}$, pendant la plus grande partie du XIX $\mathrm{X}^{\mathrm{e}}$ siècle, l'assistance aux nécessiteux n'est qu'un chapitre du maintien de l'ordre, déléguée aux communes et institutions religieuses. L'ouvrier est un libre-marchand du service «travail», mais présumé escroc et de mauvaise foi. Et pourtant, très lentement émerge le salaire indirect et ce d'abord sur l'initiative de puissantes branches industrielles (les mines, les chemins de fer) qui reçoivent la caution de l'État, et à l'usage exclusif de certaines fractions qualifiées (et dont on recherche la stabilité) de la classe ouvrière. Il existe bien entendu depuis le milieu du siècle des assurances facultatives, des fonds d'épargne, etc..., à l'usage des classes aisées. Mais ce qui est caractéristique de l'ÉtatProvidence, c'est l'obligation de cotiser et la garantie publique de la

18. Voir les «fiches historiques» de ROSANVALLON [1981], qui soulignent de façon aveuglante le lien particulier de l'État-Providence au rapport salarial et contredisent ses analyses sur leur lien au rapport marchand.

19. Nous suivons, en nous en tenant à la seule couverture sociale, la synthèse de DELORME et ANDRE qui examinent l'ensemble du rapport État-salariat (donc y compris législation, logement, école...). 
ressource. Or, même en France, ces principes ne se développent quà partir du salariat ouvrier.

Ce n'est qu'en 1898 qu'apparaît pour l'industriel l'obligation d'indemniser les accidents de travail, un fond de garantie étant créé pour parer à l'insolvabilité. La loi de retraite des cheminots est votée en 1897, mais, en 1898, il n'y a que 60000 ouvriers couverts par un système de retraite. Une première loi de généralisation et d'obligation de l'affiliation à un système de retraite porte ce chiffre à 1,6 millions en 1913. Cette loi (de 1910) fait obligation (mais sans sanction!) de cotisation pour les employeurs et les ouvriers gagnant moins de 3000 francs par an; de 3000 à 5000 francs l'affiliation est facultative. Il faut attendre l'Entre-Deux-Guerres, avec la première grande vague d'accumulation intensive, la remontée des luttes ouvtières, l'ombre de la révolution d'Octobre et... le retour à la France de l'Alsace-Lorraine, déjà dotée d'une protection sociale moderne, pour que le mouvement s'accélère un peu. 1928: création de l'Assurance-maladie obligatoire pour les salariés «sous plafond». Elle est refusée par les agriculteurs. Les assurancesretraites et accidents de travail sont un peu consolidées. Curieusement, le Front Populaire, dans sa moisson de lois sociales, n'apportera guère d'amélioration à l'État-Providence lui-même, si ce n'est la création des congés payés, forme non monétaire de salaire indirect, qui contient en germe l'idée très profonde d'une séparation de droit (et non sous la contrainte de la nécessité) entre la prestation de travail et la perception d'un revenu. Là encore, seuls les ouvriers bénéficient d'abord de cette «avancée » à vocation manifestement universelle.

C'est justement à cette époque d'affrontement direct entre patronat et ouvriers sous l'arbitrage de l'État que les classes moyennes, prestatrices réelles de services marchands (même sous la forme du salariat, comme les ingénieurs, ces «professions libérales salariées ») commencent à découvrir les avantages du sa- 
laire indirect et même du statut salariat. Comme BOLTANSKI [1982] l'analyse très finement, se développe alors un double jeu des classes moyennes: elles qui se sont toujours battues à la fois pour le maintien de la représentation «marchande» de la citoyenneté, elles qui se battent avec d'autant plus d'acharnement pour la conservation de leur autorité déléguée dans la hiérarchie sociale que celle-ci se voit court-circuitée par la grande négociation en cours, commencent à revendiquer pour elles-mêmes les avantages arrachés par la classe ouvrière. En particulier, les cadres, «bourgeois salariés», sont les mieux placés.

La primauté du salariat comme figure normative de la cohésion sociale éclate à la Libération de 1945. Les organisations ouvrières ont porté le gros du fardeau de la Résistance. D'Amérique et d'Angleterre (rapport Beveridge) parvient le modèle de la régulation monopoliste de la croissance fordiste. L'heure a sonné pour la généralisation du salaire indirect, mais alors toute les couches moyennes exigent qu'il prenne la forme d'un ÉtatProvidence couvrant l'ensemble des citoyens.

L'article 1 de l'Ordonnance de 1945 institue « une organisation de la Sécurité Sociale destinée à garantir les travailleurs et leur famille contre les risques de toute nature susceptibles de réduire ou supprimer leur capacité de gain, à couvrir les charges de maternité et les charges de familles qu'ils supportent». Mais la loi de 1946 généralise aussitôt la Sécurité Sociale et prévoit l'assujettissement obligatoire «de tout Français résidant sur le territoire français ».

En fait, de sordides marchandages aboutiront à peu près à ce résultat... sous la forme d'un éclatement inextricable de régimes particuliers ${ }^{20}$, chaque catégorie sociale préservant ses avantages acquis et, devant la poussée numérique du salariat, exigeant progressivement une «compensation démographique» [1982].

20. On en trouvera un tableau encore simplifié dans le rapport du C.E.R.C. 
pour faire financer leurs prestations sociales par d'autres (essentiellement le régime général des salariés). La création en 1958 des assurances chômages (UNEDIC), venant s'ajouter aux caisses classiques de la Sécurité Sociale (Maladie-Accident-FamilialeVieillesse) viendra tardivement compléter le dispositif, tout en renforçant ses caractères. Ceci dit, examinons donc maintenant ces caractères.

$\mathrm{Du}$ «compromis institutionnalisé » qui lui a donné naissance, l'État-protecteur à la française porte lourdement la marque.

- Il est exclusivement financé par les revenus d'activité, sous forme de cotisations versées à des Caisses sous la tutelle de l'État mais complètement distinctes du budget de l'État central. En sont donc exempts les revenus de la propriété (fermages , loyers, intérêts, dividendes, etc...) et alors que ceux-ci sont assujettis à l'impôt sur le revenu (progressif, et très faible). Les revenus de transferts (donc les retraites) sont pratiquement exempts.

- Même parmi les revenus d'activité, les agriculteurs et patrons de l'industrie et du commerce paient des cotisations relativement faibles. Certes, ils ne bénéficient pas strictement des mêmes avantages. Mais on peut dire par approximation que c'est une partie de la charge salariale des entreprises (et des administrations publiques) qui finance l'ensemble de l'ÉtatProvidence.

- Ces prélèvements sont aveugles pour le salarié. Ils prennent la forme de «cotisations employeurs» (qui n'apparaissent donc pas sur la feuille de paie) et de «cotisations salariées» qui sont directement prélevées à la source. Le salarié n'a à connaître que son salaire net. Mais pour $1 \mathrm{~F}$ de salaire net l'employeur verse en moyenne $0,65 \mathrm{~F}$ de cotisations. Ainsi est financé un budget social du même ordre de grandeur que le budget de l'Étar central sans que les «ménages» aient l'im- 
pression de sortir quelque chose de leur porte-monnaie. Forme très indolore... mais totalement opaque.

- Les prélèvements sont proportionnels aux salaires jusqu'à la limite d'un "plafond» (équivalent au salaire d'un cadre moyen ou d'un ouvrier hautement qualifié). C'est l'héritage de l'esprit «assurance» qui a présidé à la constitution de la Sécurité Sociale. Ainsi les cadres qui bénéficient de tous les avantages (familiaux, maladies) ne cotisent que sur une partie de leur salaire. Naturellement des «caisses de retraite complémentaires» à gestion paritaire ont été instituées pour assurer les retraites correspondant à la partie du salaire hors-plafond.

Ainsi, l'État-Providence est-il, du point de vue des prestations, un État des citoyens. Mais du point de vue des recettes, c'est essentiellement une collectivisation partielle du salaire, et par le jeu du plafond, majoritairement du salaire ouvrier (ou employé). On peut donc dire que les salariats ouvrier et employé co-financent l'État-Providence de tous. D'où un effet global de dépressivité des prélèvements par rapport au revenu que l'on est en droit de trouver choquant, et que ne compensent nullement la linéarité des impôts indirects par rapport à la consommation, ni la progressivité de l'impôt direct par rapport aux revenus. Les ouvriers et les employés sont imposés à près de $50 \%$ sur leurs revenus primaires, les cadres supérieurs ne le sont qu'à $42 \%$, les non salariés autour de $25 \%$.

Telle est la structure, puissante, indolore, mais injuste et fragile, qui affronte la crise du fordisme.

La crise du fordisme et la crise de l'État-Providence à la française.

Les racines de la crise du fordisme ont été analysées par ailleurs ${ }^{21}$. L'essentiel est de comprendre qu'il ne s'agit pas di-

21. Voir Aglietta [1976], Boyer et Mistral [1983], Clerc, Satre-Buisson et Lipietz [1983], Coriat [1979], Lipietz [1979, 1982], etc... 
rectement d'une crise de surproduction, comme celle de 1930 , née d'un ciseau entre les gains de productivité engendrés par l'accumulation intensive et la modération de la norme de consommation des salariés. C'est au contraire, à l'origine, une crise de rentabilité: les gains de productivité s'essouflent et ne peuvent plus compenser le maintien au rythme antérieur de la hausse du pouvoir d'achat des ménages et la hausse du coût du capital fixe. D'où une crise de l'investissement, et une tendance à remettre en cause le niveau atteint par le salaire direct. Cette pesée sur la demande finale engendre alors (mais par contre-coup seulement) le mécanisme de la spirale dépressive (croissance du chômage $\rightarrow$ baisse de la demande $\rightarrow$ baisse des embauches et de l'investissement $\rightarrow$ etc...).

Dans une telle crise, l'État-Providence joue un rôle... providentiel pour bloquer ou freiner ce «contre-coup», surtout quand est renforcé le mécanisme de l'assurance chômage ${ }^{22}$. La «demande» des ménages (de l'ordre des deux-tiers du produit marchand) se trouve en effet bloquée à la baisse. Mais il ne résoud nullement la crise à la racine, au contraire. D'une part en effet l'activité sur laquelle sont prélevées les cotisations ne progresse plus. D’autre part, les dépenses augmentent. Directement: par les allocations de chômage. Indirectement: par la «médicalisation» de l'angoisse sociale. À quoi s'ajoute la structure démographique de la France: la «catégorie» des cadres quasi-inexistante en 1945, fournit ses premiers contingents de retraités, et en outre, pour faire place au flux de jeunes entrants, on accélère les départs en retraite. D'où la déformation de la structure des dépenses en direction de ces trois postes (chômage, retraite, maladie), qui accélèrent alors que le produit national salarié stagne.

22. En 1974, l'allocation chômage des licenciés économiques cotisants de l'UNEDIC est portée à $90 \%$ du salaire antérieur, pour trois mois deux fois renouvelables... 
La solution de facilité à cette crise du financement consiste à relever les cotisations. Mais ici on se heurte à la particularité de l'État-Providence à la Française: financé sur la charge salariale des entreprises, il aggrave la crise du fordisme. Sur la plusvalue, dont la croissance est bloquée, que dévorent déjà les salaires du tertiaire improductif, et que ronge maintenant la hausse du taux d'intérêt réel ${ }^{23}$, la hausse des cotisations sociales achève d'anéantir quasiment les capacités d'auto-financement des entreprises. L'épargne brute des sociétés tombe à 10 ou $8 \%$ de la valeur ajoutée, alors que l'on considère que $7 \%$ de celle-ci représente les amortissements!

$\mathrm{Si}$ «crise de légitimité» de la Sécurité Sociale il y a, elle est d'abord là. Alors qu'en tant qu' «assurance de la capacité de dépense» elle joue macroéconomiquement un rôle stabilisateur anti-crise, par la manière spécifique dont elle est financée en France elle enfonce microéconomiquement les entreprises dans la crise. D'où la «révolte contre les charges», exprimée par les entreprises et non par les citoyens (comme en Californie ou en Suède), et d'autant plus violemment exprimée qu'il s'agit de petites entreprises dont les patrons furent et restent farouchement rétifs à l'État-Providence... en ce qui concerne les salariés.

Dans la période de la croissance, en effet, l'extension de la Protection sociale financée sur la charge salariale participait, au même titre que le jeu des prix relatifs, à un vaste mouvement de redistribution sur l'ensemble des classes sociales des gains de productivité émanant du secteur productif, plus particulièrement des industries fordistes. Plus largement, la dépense publique (dont une part, il faut le rappeler, consiste en investissements et en dépenses productives de valeurs d'usage) croissait au même rythme que le produit intérieur brut. Avec la crise, la

23. Cette hausse de la charge financière est la forme, propre à la régulation monopoliste et à sa crise inflationniste, de la dévalorisation du capital - Voir Lipietz [1983]. 
croissance de l'État-Providence creuse l'écart avec celle du produit intérieur, déplaçant de $5 \%$ l'an le partage de la valeur ajoutée brute au détriment des profits, mais pas exclusivement au profit des salariés. Du point de vue du capital comme tel, comme valeur anonyme recherchant son auto-accroissement (donc du point de vue d'A. Minc), cette évolution est intolérable. La Gauche française dans l'opposition n'en avait cure: au bout de deux ans de pouvoir, cela lui devient une priorité; il faut rétablir la capacité d'autofinancement des entreprises. Qui va payer?

C'est en fait cette question qui se cache derrière l'offensive (encore très loin d'être couronnée de succès) sur la légitimité des dépenses, alors que le débat sur la légitimité de la forme des recettes reste circonscrit à de petits cercles d'économistes proches du pouvoir. Nous y reviendrons. Restons-en ici au problème de la légitimité des dépenses.

La Protection sociale, comme organisation de flux réguliers de revenus accompagnant la croissance régulière du régime d'accumulation fordiste, se heurte donc à la crise de celui-ci sous la forme d'une barrière de financement, expression d'une remise en cause de l'évolution du partage de la valeur ajoutée. Mais, plus finement, c'est le principe de la régularité, de l'inconditionnalité de ces flux qui est remis en cause par le nécessité d'un «redéploiement», d'une restructuration du capital. En rigidifiant le revenu des salariés, l'État-Providence allège la «contrainte au travail» qui pèse sur eux. Plus généralement, les subventions aux industries obsolescentes (comme les mines et la sidérurgie), subventions à objectif social qui visent à maintenir le revenu et le statut des salariés de ces branches en les faisant travailler à perte», tombent aussi sous le coup de la critique des libéraux. Pour la droite (Bénéton), n’importe quel revenu distribué à des salariés non productivement engagés ne fait que retarder les nécessaires adaptations guidées par la «main invisible du 
marché ". C'est le fond économique qui se cache derrière l'inutile et injuste accusation de paresse adressée aux chômeurs indemnisés. Bénéton oublie qu'avant de se battre pour les indemnités, les chômeurs se battent pour trouver un emploi, et même auparavant pour garder celui qu'ils avaient, fût-ce un emploi très pénible comme celui de mineur, et dont le maintien coûte parfois plus cher à la collectivité que les indemnités de chômage correspondantes. H. Lepage se garde de l'argument moral (il n'est pas contre l'impôt négatif), mais le fond de sa critique est le même: les interventions publiques faussent le marché qui, lui, trouverait bien l'issue à la crise. Toute l'histoire économique, et en particulier l'histoire des grandes crises et de leur issue, prouve le contraire. Mais la crise du fordisme et de sa régulation monopoliste permet un retour, par la seule force du vide, des thèses libérales: tel est le fond matérialiste de la critique droitière de l'État-Providence.

Reste que le fordisme, à ses beaux jours, était en butte à une critique de gauche, en France comme partout: la critique de «l'unidimensionnalité» du double moulinet «production de masse-consommation de masse». L'État-Providence, par sa forme de salaire indirect compensatoire, contribue à l'éternisation de ce qui était alors perçu comme une aliénation. La norme, c'est de travailler dans le salariat et de consommer les produits du capitalisme. Le salaire indirect permet le maintien d'une consommation «normale» quoique réduite quand le rapport salarial est, pour des raisons indépendantes de la volonté du salarié, interrompu. L'interruption du rapport salarial doit être une interruption de toute activité productive. La croissance transitoire des besoins familiaux dûs à la production des enfants doit être couverte (outre l'accroissement du travail domestique) par des prestations monétaires prélevées sur le moulinet du capital social. Et ainsi de suite. 
Dans la période de croissance, André Gorz, et la CFDT, milieu intellectuel d'origine de P. Rosanvallon, ont particulièrement illustré la critique de ce «tout salariat» qui, avec la crise, devient de plus en plus un «tout salariat + État». Il est donc naturel qu'au sein de notre échantillon ces deux auteurs représentent une critique de l'État-Providence qui n'est que le prolongement de la critique du tout-salariat (ou du toutmarchand) de naguère. Ils représentent le refus de l'ancienne organisation fordiste du travail et de la vie quotidienne au moment où le modèle fordiste entre en crise. Critique à double destination. En exaltant les formes de créativité et de reproduction des individus et des groupes dans les marges du fordisme, au moment même où le fordisme ne peut plus assurer cette reproduction, elle peut soit offrir une «bouée de sauvetage» au fordisme (le rattrapage par la «société civile» ou «l'Économie informelle» des déchirures apparues dans la régulation monopoliste), soit au contraire appeler à un dépassement radical, noncapitaliste, du fordisme ${ }^{24}$. La «Solution» d'A. Minc au problème de la crise de l'État-Providence s'inscrit dans la première branche, le dernier ouvrage d'A. Gorz (qui implique d'ailleurs une extension absolue du revenu garanti, avec développement des formes non salariales d'expression des individus sociaux) s'inscrit dans l'alternative. P. Rosanvallon penche plutôt vers la problématique d'A. Minc.

En guise de conclusion: quelques pistes pour une réforme progressiste de l'État-Providence

Chemin faisant, nous avons donc pu rapporter les critiques de l'État-Providence (telles qu'elles s'expriment dans l'échantil-

24. Face à la montée des thèses illichiennes, écologistes, anti-productivistes, au moment même de l'entrée en crise du fordisme, j'ai moi-même hésité sur la caractérisation conjoncturelle de ce type de critique. Voir par exemple mes articles des Temps Modernes, Décembre 1976, Juin 1980, etc..., avec leur difficile rapport à l'évolution d'André Gorz. 
lon que nous avons choisi) à la crise objective de l'ÉtatProvidence, comme composante de la crise du fordisme, avec sa spécificité française. Il nous reste à évoquer brièvement ce que pourrait être un dépassement «progressiste» de l'actuel ÉtatProvidence. Naturellement, cette analyse n'ayant pas la prétention d'être une contribution à l'élaboration d'un programme politique, il ne s'agit pas d'énoncer ce que l'auteur, subjectivement, considère comme juste ou simplement réaliste. Il s'agit simplement, à partir de l'interprétation proposée dans la seconde partie, et à partir de l'analyse des critiques présentées dans la première, d'indiquer un certain nombre d'axes de réflexion absents dans les critiques analysées, ou de resituer dans une autre perspective certaines propositions évoquées.

Car, malgré la légitimité dont jouit l'État-Providence dans «l'opinion publique» telle que la met en forme les sondages, sa crise ne peut manquer de s'amplifier d'ici quelques années, ne serait-ce que sous la pression du déficit. S'exprimeront alors violemment des points de vue des différentes classes et groupes sociaux, avec des arguments d'un tout autre style que celui des «pamphlets savants».

\section{Ce que ne devrait pas être le débat sur l'État-Providence.}

Dans les critiques initialement présentées, nous avons perçu certains biais dûs à une insuffisance d'analyse de la réalité de l'État-Providence à la française. Sont ainsi mélangées à des critiques parfois pertinentes des critiques, qui peuvent par ailleurs renvoyer elles-mêmes à de véritables problèmes, mais qui ne devraient pas s'adresser spécifiquement à l'État-Providence.

Ainsi, on critique (chez Rosavallon, ou Gorz) l'ÉtatProvidence comme forme monétaire de revenu destiné à couvrir des besoins, alors qu'en fait on vise le principe même de la reproduction salariale ou marchande. Que des besoins puissent 
être satisfaits autrement que par l'achat des biens et de services produits par le capitalisme (ou la sphère «hétéronome»), qu’il y ait une aspiration sociale à la satisfaction autonome de ces besoins, c'est certainement vrai. Mais la satisfaction autonome des besoins s'oppose à la satisfaction marchande des besoins, quelle que soit la forme des revenus monétaires couvrant ces dépenses: salaire direct ou indirect. La critique de la "société marchande» s'adresse autant au "tout salariat» qu'au «tout à l'État». La dynamique sociale s'orientera peut-être vers un élargissement de la sphère autonome, mais resteront encore longtemps des rapports marchands. Dans la sphère circonscrite de la reproduction marchande, le débat sur l'État-Providence se limite alors au partage entre revenu direct d'activité et revenu indirect de transfert.

De la même manière, il est abusif d'imputer l'ÉtatProvidence «à la charge» de la Société. L'État-Providence tel que nous l'avons défini est un État redistributeur: il redistribue entre les individus des masses de valeur qui sont déjà affectées aux revenus des ménages, et pour la masse de ceux-ci la redistribution est à somme nulle (aux coûts de gestion près). Si toutes les cotisations étaient reversées aux revenus directs, chaque ménage serait d'un coup plus riche mais devrait assumer directement plus de dépenses, et faire plus d'économies. La plupart auraient recours à un système d'assurance privé qui lui aussi aurait son coût, tout aussi contestable et sans doute plus élevé que celui de la gestion publique (voir l'exemple des compagnies d'assurance). En revanche, il resterait aussi peu pour l'accumulation productive. Il y a un vrai débat sur le partage entre revenus des ménages et autofinancement des entreprises. Il doit être traité comme tel. Mais si l'on estime qu'il faut accroître l'autofinancement, c'est la distribution de l'ensemble des revenus qui est en cause: on s'aperçoit alors que la Protection sociale exerce une ponction du secteur non salarial sur l'ensemble «entreprises 
+ salariés». C'est la seule «charge» spécifique de l'ÉtatProvidence sur «l'économie», et elle est spécifique au «compromis institutionnel» français.

Plus précisément, l'ampleur des inégalités sécrétées par ce compromis rend dérisoire le débat sur «l'excès d'égalité » qu'engendrerait l'État-Providence. On peut considérer les inégalités de revenu comme légitimes: c'est un débat différent. Mais à partir du moment où l'on considère que l'État-Providence couvre l'ensemble des citoyens, c'est à la mesure de leurs inégaux revenus et non de leurs statuts différents qu'ils devraient y contribuer. Tel est le véritable débat sur l'efficience redistributive du système.

Enfin, le débat porté par les nouveaux économistes sur l'efficience économique et la liberté est particulièrement équivoque. Rappelons que, sauf l'assurance maladie et en un sens les allocations familiales qui affectent les revenus de transfert à une dépense particulière (et qui mériteraient donc une discussion particulière), tous les autres revenus de transferts laissent une totale «liberté du consommateur» à leurs bénéficiaires. Que ces revenus ne compensent pas correctement l'effort fourni est un jugement de valeur totalement subjectif à prétention totalitaire sur le «pretium doloris» de l'angoisse du chômage, de la fatigue du vieux travailleur, de la faiblesse du convalescent, etc... Tout aussi subjective et totalitaire est l'affirmation selon laquelle les transferts encouragent «trop» les loisirs et découragent du travail. Si la société juge qu'elle devrait davantage travailler contre monnaie et moins faire de cuisine, de sport, ou de piano, c'est un choix de société, non un problème d'efficience. Il y a en revanche un véritable problème, celui de la transparence de ce choix, qui se décompose lui-même en trois aspects, déjà évoqués:

- le partage activité autonome/activité salariale (ou marchande) 
- dans l'activité salariale-marchande, le partage accumulation/revenu des ménages

- dans les revenus des ménages, le partage entre revenus directs (d'activité) et indirects (de transfert).

Ces choix sont d'une immense ampleur, et concernent la société dans sa capacité à s'instituer à travers les conflits et les compromis sociaux.

Ils débordent de toute part celui des modalités concrètes de ce "résidu», l'organisation publique des revenus de transferts, à quoi nous avons limité l'acception du terme "ÉtatProvidence ». Certes, le débat concret sur l'État-Providence les mettra tous en jeu. Mais qu'au moins on mesure ce qui est débat général sur le partage social du temps, et ce qui est débat spécifique à l'État-Providence à la française!

\section{Le grand débat: la remise en cause du compromis de 1946.}

Actuellement se dessine en France un assez large consensus implicite pour:

- rétablir la capacité d'autofinancement des entreprises

- stopper la progression du pouvoir d'achat des revenus directs

- diminuer progressivement la durée du travail hebdomadaire, et la durée de vie salariée (l'abaissement à 60 ans de l'âge de la retraite est une des mesures les plus populaires du gouvernement de gauche)

- ne pas remettre en cause les prestations indirectes acquises, et les étendre progressivement (les agricultrices viennent enfin d'obtenir des «congés» de maternité).

Ces choix sont implicites, mais à eux seuls, dans un contexte de croissance ralentie, ils impliquent une accélération de la part des transferts. Or, on l'a vu, ces transferts sont en France prélevés sur la masse salariale des entreprises, ce qui est contradiction avec le premier objectif: on va vers la «crise des 
charges des entreprises» qui, avec la «contrainte extérieure», est en fait le grand problème économique de l'heure. De toute façon le problème du financement de l'État-Providence est posé.

Augmenter la part des cotisations «employeurs» (ce qui revient à déplacer le partage de la valeur ajoutée au détriment des profits) est définitivement exclu. Augmenter la part des corisations «employés» est politiquement difficile; la gauche peut imposer une stagnation du pouvoir d'achat aux travailleurs, pas un recul, ou du moins pas à eux seuls. La question de l'«équité de la pression contributive» est donc posée. Derrière elle se profile celle de la distribution des revenus primaires et finaux (une des plus inégalitaires de l'OCDE), mais il est probable que le débat se restreindra d'abord à l'équité des transferts réalisés par la Sécurité Sociale.

Or nous avons vu que le compromis de 1946, non seulement n'était pas redistributif (des riches vers les pauvres), mais encore accentuait l'inégalité des classes sociales. Du côté des recettes: prélèvements sur le revenu de la propriété nuls, faibles pour les profits d'entreprises individuelles, plus faibles en proportion sur le salaire des cadres que sur celui des exécutants. Mais il en est de même du côté des dépenses.

Ainsi, par le jeu des durées de vie (on meurt tôt dans la classe ouvrière) et du plafonnement, les cadres récupèrent une fois et demi leurs cotisations de retraite, les ouvriers ne font que récupérer (statistiquement!) leur «salaire indirect». Et ne parlons pas des non-salariés (voir tableau 1).

De même, la politique familiale passe principalement par la «dépense budgétaire», sous forme d'un «quotient familial» servant au calcul des impôts directs. Ceux-ci étant assez progressifs, les enfants (ou les épouses!) des tiches sont «subventionnés» considérablement plus que ceux des pauvres (de 2 à 10 fois plus entre manœuvres et cadres supérieurs). Enfin, il est 
TABLEAU 1

Bilan redistributif des retraites *

\begin{tabular}{lrrrr}
\hline & $\begin{array}{c}\text { Pensions } \\
\mathrm{P}\end{array}$ & $\begin{array}{c}\text { Cotisations } \\
\mathrm{C}\end{array}$ & $\mathrm{P}-\mathrm{C}$ & $\mathrm{P} / \mathrm{C}$ \\
\cline { 2 - 5 } & & & & \\
\cline { 2 - 5 } & & & & \\
Classes hommes du « Privé » & 929,120 & 590,280 & 338,830 & 1,57 \\
$\quad$ Cadres supérieurs & 502,700 & 349,670 & 153,030 & 1,44 \\
Cadres moyens & 267,430 & 219,220 & 58,210 & 1,28 \\
Employés & 412,080 & 322,150 & 89,930 & 1,28 \\
Contremaîtres & 242,520 & 214,660 & 27,870 & 1,13 \\
Ouvriers qualifiés & 221,510 & 198,510 & 23,000 & 1,12 \\
Ouvriers spécialisés & 157,150 & 150,680 & 6,470 & 1,04 \\
Manouvres & 248,080 & 172,810 & 75,270 & 1,43 \\
Personnels de service & 179,950 & 171,790 & 8,160 & 1,05 \\
\hline Salariés agricoles & 120,360 & 24,380 & 95,980 & 4,94 \\
\hline Exploitants agricoles & & & & \\
\hline
\end{tabular}

* Il s'agit d'un bilan sur la durée de vie des prestations reçues et des cotisations versées. Ce bilan est établi sur la base de la législation de 1976.

Source: J. P. Launay. Modèle BABAR. Direction de la Prévision.

connu que la consommation médicale est d'autant plus élevée que les revenus le sont ${ }^{25}$.

«Décharger les entreprises» et répartir plus équitablement le fardeau exige une extension générale de l'assiette des prélèvements de la masse salariale (où ils sont essentiellement concentrés) à l'ensemble du revenu national, ou au moins à l'ensemble du revenu des ménages, soit sous la forme de l'impôt sur le revenu, soit sous la forme d'impôt indirect sur la consommation. Alors seulement la Protection sociale deviendra ce qu'elle prétend être, et qu'elle est devenu seulement du côté des dépenses: une solidarité entre citoyens, et non plus un salaire indirect pillé par les non-salariés. Citons un seul chiffre: le financement

25. Il existe de multiples études sur ces sujets. Voir BLUM-GIRARDEAU [1981], CERC [1982], ou le rapport du CREDOC: "Les prestations-maladie par catégoric de famille» de Hatchuel et Mannoni (Décembre 1982). 
des allocations familiales, actuellement assuré par les employeurs à raison de $9 \%$ de la masse salariale, exigerait un impôt linéaire sur l'ensemble des revenus de $6 \%$ seulement. Ce qui laisserait (en supposant que les entreprises transferent $6 \%$ au salaire direct, ce qui serait une opération blanche pour les salariés sous plafond) 3\% d'Épargne Brute supplémentaire: soit une hausse de $30 \%$ de la capacité d'autofinancement des entreprises.

Naturellement, la fiscalisation complète, par l'impôt sur le revenu progressif, de toute la sécurité sociale, qui se traduirait par un décuplement de l'impôt direct et un repartage général du revenu des classes sociales au bénéfice des ouvriers et des employés, est totalement impensable en l'état actuel du rapport des forces entre les classes en France. Elle pourrait cependant s'étendre progressivement, avec une généralisation du prélèvement à la source, et combinée aux impôts indirects, en commençant par le financement des prestations les plus ouvertement «universalistes», la famille et (ce qui est un beaucoup plus gros morceau) la santé ${ }^{26}$. En revanche, les cotisations de chômage et de retraite, qui ressemblent le plus à une forme d'épargne de précaution organisée, modulant le revenu de la vie active sur l'ensemble de l'existence, semblent n'appeler (du côté des recettes) que des modifications techniques, alignant les cotisations sur l'espérance de vie à la retraite, et faisant davantage contribuer ceux qui risquent le moins le chômage.

\section{Du côté des dépenses: des problemes spécifiques.}

Nous avons remarqué au passage que les prestations sociales se divisaient en deux groupes: celles qui couvrent les dépenses précises (famille, soins médicaux) et celle qui assurent un

26. Le gouvernement de gauche s'oriente dans ce sens: déclaration d'intention en ce qui concerne les allocations familiales, et décisions ponctuelles de fiscalisation (handicapés, avortements...). En Mars 83 , un impôt linéaire de $1 \%$ sur le revenu est institué, destiné prioritairement à la Caisse d'Allocation Familiale. 
revenu permanent par-delà les aléas (chômage, retraite, indemnisation des congés médicaux). C'est par rapport à cette diversité que doivent être abordées les questions des «économies» ou encore de la «substitution de l'autonomie à l'assistance», deux notions dangeureusement confondues... Derrière ces questions se cachent les rapports: marchands/non marchands, activité/loisir, etc...

Voyons d'abord les dépenses «affectées». Ce sont les seules par lesquelles l'institution «État-Providence» semble restreindre la liberté des choix et l'initiative du citoyen, c'est par elles que passe la «pression sociale» sur la structure de consommation des individus. Dans le cas des allocations familiales, il s'agit d'un véritable choix social, très centralisé, d'inspiration nataliste. On peut discuter de son efficacité positive. Ce qui est certain, c'est que leur suppression rendrait encore plus difficile d'avoir des enfants, car il est connu que le revenu par tête d'un ménage dépend, au moins autant du revenu du chef de ménage que du nombre d'enfants. Sauf à pénaliser les familles, les «économies» ne peuvent venir que d'une collectivisation microsociale de certaines activités domestiques, qui risque de se solder par un transfert de charges en direction du travail féminin gratuit...

Du côté des dépenses de santé, la comparaison de l'Angleterre (fonctionnariat et médecine gratuite), de la France (médecine libérale et remboursement des soins, librement choisis, par une assurance publique) et des USA (médecine libérale avec assurance semi-facultative) montre que, dans les pays à revenus élevés, plus les gens peuvent «librement choisir», plus «les consommateurs sont les payeurs", et plus est forte la part des dépenses de santé dans le revenu national: aux USA, suivis par la France puis la Grande-Bretagne.

Inutile d'incriminer par conséquent la « déresponsabilisation» du patient: s'il avait à choisir, il paierait encore plus, et 
de sa poche, à condition de le pouvoir. "Responsabiliser» le malade (tickets modérateurs, assurance complémentaire...) ne ferait qu'engendrer une médecine à deux vitesses, sans probablement diminuer les dépenses. Reste le problème de la responsabilisation de l'offre: le complexe «industrialo-hospitalier" qui, pesant de tout son poids sur «l'aiguilleur» que constitue le médecin généraliste, est le destinataire final des dépenses de la Caisse Maladie ${ }^{27}$. Il s'agit donc d'un ensemble de productions marchandes dont la validation sociale est automatiquement assurée! D'où une progression de l'ordre de $20 \%$ l'an, qui traduit une tendance à la médicalisation des problèmes sociaux, à la spécialisation des problèmes médicaux, à l'hospitalisation des problèmes spéciaux...

Il ne saurait être question, au nom des nécessaires économies, d'entonner le grand air de la "contreproductivité» de la médecine moderne. Depuis la Seconde Guerre mondiale, la médecine devenue gratuite a quasiment vaincu la mortalité infantile, et si $50 \%$ des dépenses de santé concernent $4 \%$ de malades dont la moyenne d'âge est 65 ans, c'est le prix qu'il a fallu payer pour prolonger de 10 ans la vie humaine, et sans doute davantage la vie «non-grabataire». Reste que les stricts mécanismes institutionnels poussent à la dépense bien au-delà de l'utile. La seule «déshospitalisation» de la médecine permettrait déjà des économies qui risquent, encore une fois, de retomber sur le travail domestique gratuit des femmes.

Dans le cas de la maladie et de la famille, il semble donc que le maintien du «niveau de service» ne puisse autoriser des économies de dépenses que par un transfert au non-marchand, ce qui peut avoir des avantages pour la qualité de la vie individuelle et sociale, à la condition absolue que ce retrait de la

27. Sur les déterminants des dépenses de santé, voir SAILLARD [1979]. 
Providence monétaire ne soit pas payé par un retour en force de l'exploitation ancestrale des femmes au foyer.

Le problème des retraites, du chômage (et on pourrait y ajouter des stages de formation des jeunes) est totalement différent. Il en serait de même pour la Prime aux Volontaires du Travail à Temps réduit de Michel Albert. Ici, on se rapproche de la notion de revenu garanti décuplé de l'offre d'emploi. Mais l'esprit en reste encore très loin du «revenu social» d'André Gorz.

Tout d'abord, ces revenus resteront encore longtemps des revenus de substitution à des revenus d'activiré, situation de référence «normale» qui en détermine largement le montant. Dans un pays qui est une mosaïque de corporations et de catégories plus jalouses même de leurs privilèges différentiels que du niveau absolu de leurs revenus, on voit mal comment il pourrait en être autrement: la hiérarchie des revenus se détermine dans la lutte des classes et des catégories, principalement dans l'entreprise.

En revanche, on peut envisager d'en finir avec l'association kafkaïenne du revenu de transfert avec l'obligation sociale de non-activité. Tout se passe comme si les transferts (chômage, pré-retraite) étaient de plus en plus le prix payé aux individus pour qu'ils dégagent le marché du travail. S'il s'agit de constater que la sphère des activités marchandes concurrentielles est contrainte par la crise mondiale, cela peut être légitime. Mais il n'y a aucune raison d'interdire toute activité créatrice de richesses matérielles ou intellectuelles aux «exclus» (ou dispensés?) du travail salarié. Cette exclusion est renforcée par le découpage de la vie humaine en tranches absurdes: formation-travail (haché de chômage-retraite. Résultat: ceux qui travaillent «perdent leur vie à la gagner» à l'âge où ils sont les plus imaginatifs et disponibles aux réorientations, ceux qui vivent de transferts et ne font rien sont socialement considérés comme des assistés, 
ceux qui vivent de transferts et ont une activité sont considérés comme des cumulards voire des escrocs... Ne serait-il pas plus sage, selon la ligne d'André Gorz, de considérer les revenus de transferts prélevés sur la sphère de l'activité «formelle» (généralement: le salariat) comme une forme de subvention marchande à des périodes d'activité «différente», à répartir dans la durée de la vie humaine, où chacun pourrait choisir entre le loisir, la création artistique, le recyclage professionnel, l'expérimentation de nouvelles formes de production, marchandes ${ }^{28}$ ou bénévoles, etc...? Irait dans ce sens l'ouverture des droits à la retraite après un nombre donné d'années de travail salarié, des «années sabbatiques» librement choisies étant couvertes au même niveau, et décalant d'autant l'âge du départ en retraite. Irait également dans ce sens l'affectation des fonds du chômage à des expériences de créations d'emploi coopératifs ou d'utilité sociale, etc... ${ }^{29}$. Comme on le voit, il existe des cheminements raisonnables dans la voie de l'Utopie...

\section{Bibliographie}

AGLIETTA, M. [1976]

Régulation et crises du capitalisme, Calmann-Lévy, Paris.

ALBERT, M. [1982]

Le pari français. Le nouveau plein emploi, Seuil, Paris.

BENETON, P. [1983]

Le fléau du bien. Essai sur les politiques sociales occidentales, R. Laffont, Paris.

28. Ce qui pose le problème de la «juste concurrence» entre le travail informel-marchand ainsi «blanchi" et la petite production marchande artisanale «régulière». Cela peut passer par une modification du régime fiscal des artisans, mais implique une répression accrue du salariat clandestin.

29. Il arrive aujourd'hui que les indemnités de chômage soit débloquées d'un coup pour six mois à des collectifs de travailleurs décidant de reprendre à leur compte, sous forme de coopérative, leur entreprise en faillite. La Caisse d'Assurance Chômage fait alors simplement le pari économique que la coopérative vivra plus de six mois... Mais c'est une manœuvre à la limite de l'actuelle légalité, et il est encore plus rare de créer des emplois ex-nihilo avec les allocations de chômeurs décidant de se regrouper pour entreprendre quelque chose. 
BLUM-GIRARDEAU, C. [ 1981]

Les tableaux de la solidarité, Èconomica. La documentation française, Paris.

BOLTANSKI, L. [1982]

Les Cadres, Minuit, Paris.

BOYER, R., MISTRAL, J. [1983]

Accumulation, inflation, crises, $2^{\mathrm{e}}$ édition refondue, P.U.F., Paris.

de BRUNHOFF, S. [1971]

L'offre de monnaie, Paris, Maspéro.

C.E.R.C. [1982]

Comparaison des régimes de sécurité sociale, La Documentation Française, Paris.

CLERC, D., LIPIETZ, A., SATRE-BUISSON, J. [1983]

La crise, Syros, Paris.

CORIAT, B. [1979]

L'atelier et le chronomitre, Bourgois, Paris.

DELORME, R., ANDRE, C. [1983]

L'État et l'économie, Seuil, Paris.

DUMONT, L. [1977]

Homo Aequalis, Gallimard, Paris.

DUPEYROUX, J.J. [1983]

«La délation. Un point de vue sur le travail noir», Le Monde, 19 janvier.

GORZ, A. [1980]

Adieux au prolétariat, Galilée, Paris.

GORZ, A. [1983]

Les chemins du Paradis. L'agonie du capital, Galilée, Paris.

GREFFE, X. [ 1983]

«L'assurance, c'est la société duale», Inter-Social n ${ }^{\circ} 89$, Mars.

LAFAY, J. D. [1983]

"Les effets - prix de la protection sociale», Analyses de la SEDEIS, Janvier.

LEPAGE, M. [ 1978]

Demain le capitalisme, Librairie Générale Française, Paris.

LEPAGE, M. [1980]

Demain le libéralisme, Librairie Générale Française, Paris.

LINHART, D. [1981]

L'appel de la sirène, Sycomore, Paris.

LIPIETZ, A. [ 1979]

Crise et Inflation: pourquoi?. Paris, F. Maspéro. 
LIPIETZ, A. [ 1982]

"Derrière la crise: la tendance à la baisse du taux de profit», Revue Économique $\mathrm{n}^{\circ}$ 2, Mars.

LIPIETZ, A. [1983A]

Le Monde Enchanté. De la valeur à l'envol inflationniste, La DécouverteMaspéro, Paris (à paraître en septembre).

LIPIETZ, A. [1983B]

"Les transformations dans la division internationale du travail», intervention au Colloque Le Canada et la Nouvelle Division Internationale du Travail, Ottawa, 28-29-30 Janvier 1983, miméo CEPREMAP 8302.

MARX, K. [1867] Le Capital, Livre I, Garnier-Flammarion, Paris.

MEILLASSOUX, C. [1975]

Femmes, greniers, et capitaux, F. Maspéro, Paris.

MINC, A. [1982]

L'après-crise est commencé, Gallimard 1982.

PARKER, H. [1982]

«The moral hazard of social benefits: a study of the impact of social benefits and income tax on incentives to work", Research Monograph, $\mathrm{n}^{\circ}$ 37, Institute of Economic Affairs, London.

ROSANVALLON, P. [1980]

«Le développement de l'économie souterraine et l'avenir des sociétés industrielles», Le Débat $\mathrm{n}^{\circ} 2$, Juin.

ROSANVALLON, P. [1981]

La crise de l'État-providence, Seuil, Paris.

ROSANVALLON, P. [1983]

Misère de l'économie, Seuil, Paris.

ROWBOTHAM, S., SEGAL, L., WAINWRIGHT, H. [ 1980]

Beyond the fragments. Merlin Press, London.

SAILLARD, Y. [ 1979]

"Croissance des dépenses de santé: quelques éléments pour une approche en terme de régulation», Journées d'Économie sociale, CNRSParis X, miméo, Juin.

STOLERU, L. [1977]

Vaincre la pautreté dans les pays riches, Flammarion, Paris. 